\title{
Correction
}

\section{Correction to: Cross-projects software defect prediction using spotted hyena optimizer algorithm}

\author{
M. A. Elsabagh ${ }^{1}$ M. S. Farhan ${ }^{2}$ - M. G. Gafar ${ }^{3,1}$
}

Published online: 31 January 2022

(c) Springer Nature Switzerland AG 2022

Correction to: SN Applied Sciences (2020) 2:538

https://doi.org/10.1007/s42452-020-2320-4

In this article, affiliation no. 1 incorrectly showed as the

Publisher's Note Springer Nature remains neutral with regard to last author's first affiliation. The original article has been jurisdictional claims in published maps and institutional affiliations. corrected.

The original article can be found online at https://doi.org/10.1007/s42452-020-2320-4.

M. A. Elsabagh, Mahmoud_Mohsen@fci.kfs.edu.eg; Elsapgh2010@gmail.com | Department of Machine Learning and Information Retrieval, Faculty of Artificial Intelligence, Kafrelsheikh University, Kafr Elsheikh, Egypt. ${ }^{2}$ Department of Information Systems, Faculty of Computers and Artificial Intelligence, Helwan University, Cairo, Egypt. ${ }^{3}$ Department of Computer Science, College of Science and Humanities in Al-Sulail, Prince Sattam Bin Abdulaziz University, Kharj, Saudi Arabia. 